\title{
miR-136 suppresses tumor invasion and metastasis by targeting RASAL2 in triple-negative breast cancer
}

\author{
MEISI YAN, XIAOBO LI, DANDAN TONG, CHANGSONG HAN, RAN ZHAO, YAN HE and XIAOMING JIN \\ Department of Pathology, Harbin Medical University, Harbin, Heilongjiang 150081, P.R. China
}

Received November 20, 2015; Accepted December 17, 2015

DOI: $10.3892 /$ or.2016.4767

\begin{abstract}
MicroRNAs play an important role in the regulation of cancer migration, invasion and metastasis. Patients with triple-negative breast cancer (TNBC) have a high incidence of early relapse and metastasis; however, the molecular basis for metastasis and recurrence in these individuals remains largely unknown. Herein, we demonstrate that miR-136 is an anti-invasive microRNA in TNBC and suppresses mesenchymal invasion and metastasis. Our results demonstrated that miR-136 was downregulated in TNBC and negative correlated with the WHO grades. However, RASAL2 was identified as a functional target of miR-136, and was overexpressed in TNBC and correlates with pathological grades. Moreover, overexpression of RASAL2 in a breast cancer cell line rescued miR-136-mediated cell migration and invasion. In conclusion, these results indicate that the miR-136/RASAL2/MET axis act as a suppressor of TNBC metastasis.
\end{abstract}

\section{Introduction}

Breast cancer is the most frequently diagnosed female cancer worldwide and remains the top cause of cancer death in females, although the diagnostic techniques and treatment modalities advanced greatly $(1,2)$. Triple-negative breast cancer (TNBC) is a heterogeneous group of breast cancers, characterized by the loss of estrogen receptor (ER), progesterone receptor (PR) and human epidermal growth factor receptor 2 (HER2) gene expression (3). As the representative of the most aggressive subtype, it accounts for $10-20 \%$ of all invasive breast cancers (4). Since there is no targeted therapy available at present, one-third of patients with this disease develop recurrence within 3 years, even when receiving adjuvant therapy $(5,6)$. Hence, understanding the mechanisms underlying distant metastasis and early relapse in TNBC will

Correspondence to: Professor Xiaoming Jin or Professor Yan He, Department of Pathology, Harbin Medical University, Baojian Road 157, Nangang, Harbin, Heilongjiang 150081, P.R. China

E-mail: jinxm55@163.com

E-mail: he_yan419@163.com

Key words: triple-negative breast cancer, miR-136, RASAL2, invasion, metastasis be key to discover new therapeutic targets and improve clinical treatment of these patients.

The Ras pathway is one of the most commonly deregulated pathways in human cancer (7), as one-third of human cancers have been observed to harbor high frequency of mutational activation of Ras proteins (8). Ras proteins, together with guanine nucleotide exchange factors (GEFs) and GTPaseactivating proteins (GAPs), constitute cellular binary switches cycling between 'on' and 'off' conformations determined by the loading of GTP or GDP, respectively. GEFs stimulate GDP for GTP exchange, and therefore activates the Ras pathway, whereas GAPs terminates the activation status by promoting GTP to GDP hydrolysis (9). RASAL2, function as a GAP, and has been shown to be implicated in the establishment and metastasis of several types of tumors, such as lung, ovarian, thyroid and breast cancer (10-13). A recent report showed that RASAL2 was oncogenic in TNBC and drives mesenchymal invasion and metastasis. Moreover, RASAL2 expression was tightly associated with the poor prognosis of patients suffering of TNBC (14).

MicroRNAs (miRNA) are a class of small, endogenous, non-coding RNAs that regulate gene expression post-transcriptionally (15). Accumulating studies have demonstrated that miRNA-regulated transcriptional dynamics was a critical step in tumor initiation, promotion and progression $(16,17)$. In human cancers, many miRNAs were identified to function as potential tumor suppressors and their downregulation leads to overexpression of oncogenic genes (18-20). miR-136 was recently identified to be tightly associated with tumorigenesis and metastasis. It was first characterized to be upregulated in human and murine lung cancers by miRNA microarray expression profiling (21). miR-136 was also reported to be predominately overexpressed in the Jurkat cell line (22) and was found to target tumor suppressor PTEN in breast cancer cells (23), indicating a possible significance in cancer development and progression. Further studies showed that it may act as a cancer promoting gene in human non-small cell lung cancer (NSCLC) (24) and it was found to be downregulated in human glioma and promotes apoptosis of glioma cells by targeting AEG-1 and Bcl-2 (25), indicating it may also act as an tumor suppressor. The precise role of miR-136 in breast cancer, especially in TNBC, remains largely unknown. In the present study we report that miR-136 may act as an tumor suppressor in TNBC. Decreased expression of miR-136 was observed in TNBC correlating with the pathological grades, 
and overexpressed miR-136 restrained the migration and invasion of MDA-MB-231 cells (a highly invasive TNBC cell line). We further demonstrated that the anti-invasive effect of miR-136 was mediated through targeting RASAL2, a cancer-promoting gene in TNBC. Our results demonstrated that miRNA-136 was a key anti-invasive miRNA and further confirmed the oncogenic role of RASAL2 in TNBC.

\section{Materials and methods}

Cell culture, plasmids and transfection. MCF10A, MCF7, ZR751 and MDA-MB-231 cells were cultivated in Leibovitz's L-15 Medium containing 10\% fetal bovine serum plus $2 \mathrm{mM}$ L-glutamine. The cells were split before confluence and incubated at $37^{\circ} \mathrm{C}$ in a humidified incubator with $5 \% \mathrm{CO}_{2}$. miR-136 mimics and antisense oligonucleotides (ASO) were purchased from Shanghai GenePharma Co., Ltd. (Shanghai, China). RASAL2 full length CDS were cloned to pCMV2myc. Transfection of miRNA mimics and ASO was carried out using Lipofectamine 2000 (Invitrogen, Carlsbad, CA, USA) according to the manufacturer's instructions.

Human tissue samples. Forty TNBC samples or adjacent normal mucosa tissues were obtained from patients with TNBC. Detailed pathological and clinical data were collected for all samples including WHO tumor grade, invasion and metastasis. The diagnoses of these samples were verified by pathologists. The collection of human tissue samples was approved and supervised by the Ethics Committee of Harbin Medical University.

Mouse xenograft model. Female immune-deficient nude mice (strain BALB/c nu/nu; 4-5 weeks old) used in the present study were bred at the Department of Pathology, Harbin Medical University. For orthotopic injection, $1 \times 10^{7}$ MDA-MB-231 cells were injected into the mammary gland fat pad of each CB-17 SCID mouse in a volume of $0.1 \mathrm{ml}$. miR-136 mimics and its negative control miRNA were injected into the tumor every 2 days from day 7 of the graft. Twenty-one days later, the tumors were collected and sectioned for immunohistochemical analysis.

Immunohistochemistry analysis. Tumor tissues of xenograft mice, human colon cancer samples or adjacent normal mucosa tissues were fixed in $10 \%$ neutral buffered formalin for $24 \mathrm{~h}$ and then embedded in paraffin. Sections $(4 \mu \mathrm{m})$ were cut and stained for histological examination. E-cadherin (BD Biosciences; cat. BD 610182), RASAL2 (Santa Cruz Biotechnology; cat. sc-67935) antibody was diluted in PBS with 1\% (wt/ vol) BSA. Images were obtained with a Nikon DP70 camera mounted on a Nikon Bx60 microscope with Cell-F imaging software (Soft Imaging System).

RNA preparation and quantitative PCR. RNA was extracted from cells or tissue samples using the mirVana miRNA isolation kit (Ambion, Foster City, CA, USA) according to the manufacturer's instructions. Small RNA fraction $(<200 \mathrm{nt})$ was separated and purified according to the procedure. cDNA was obtained using M-MLV (Promega, Madison, WI, USA) and $1 \mu \mathrm{g}$ RNA. The relative level of miR-136 was detected by stem-loop RT-PCR with the following conditions: denaturing the DNA at $94^{\circ} \mathrm{C}$ for $4 \mathrm{~min}$, followed by 40 cycles of amplification: $94^{\circ} \mathrm{C}$ for $60 \mathrm{sec}, 58^{\circ} \mathrm{C}$ for $60 \mathrm{sec}, 72^{\circ} \mathrm{C}$ for $60 \mathrm{sec}$ for data collection. U6 snRNA was used as an endogenous control. Quantitative PCR was performed on an ABI 7500 thermocycler (Applied Biosystems) using SYBR ${ }^{\circledR}$ Premix Ex $\mathrm{Taq}^{\mathrm{TM}}$ (perfect real-time) kits (Takara Bio, Inc., Shiga, Japan) according to the manufacturer's instructions.

Wound-healing assay. Equal number of MDA-MB-231 cells transfected with miR-136 mimics and control miRNA were seeded on BioCoat ${ }^{\mathrm{TM}}$ collagen I coated 6-well tissue culture dishes (BD Biosciences; cat. 354400) respectively, and allowed to grow to confluent for $48 \mathrm{~h}$. Then scratches were made using p200 pipette tips, however, floating cells were carefully washed away with fresh growth medium. The wound-healing of the scratch regions were monitored and imaged at designated time-points.

Cell migration and invasion assays. A total of $5 \times 10^{4}$ MDA-MB-231 cells (in 0.2 ml RPMI-1640 with 5\% FBS) were seeded into the upper part of a Transwell chamber (Corning Life Sciences, Corning, NY, USA). For migration invasion assay, the chamber was pre-coated with $1 \mathrm{mg} / \mathrm{ml}$ Matrigel (Growth Factor Reduced BD Matrigel ${ }^{\mathrm{TM}}$ Matrix) for $2 \mathrm{~h}$. In the lower part of the chamber, $0.6 \mathrm{ml}$ RPMI-1640 with $20 \%$ FBS was added. After incubating for $30 \mathrm{~h}$, chambers were disassembled and the membranes were stained with $2 \%$ crystal violet for $10 \mathrm{~min}$ and placed on a glass slide. Then, cells on the bottom of the membranes were counted in 5 random visual fields under a light microscope. All assays were performed in triplicate and independently performed three times.

Fluorescent reporter assays. The human RASAL2 3'UTR harboring three miR-136 potential target-binding sequences was synthesized by Shanghai GenePharma. Luciferase constructs were made by ligating the synthesized 3'UTR as well as the seed-sequence mutated version after the lucORF in the pMIR-Report luciferase vector (Ambion). For the fluorescent reporter assay, cells were seeded in a 48-well plate the day before transfection. The cells were co-transfected with miRNA mimics or ASO as well as the controls and RASAL23UTR or RASAL2-3UTRmut. The cells were lyzed $48 \mathrm{~h}$ later and the intensity of luciferase was detected.

Western blotting. Western blotting was performed to determine protein expression and the GAPDH was used as the internal control. Total protein from cells were lysed by RIPA buffer and then measured by Micro BCA protein assay kit (Pierce Biotechnology). Protein (50 $\mu \mathrm{g} /$ lane) was resolved on sodium dodecyl sulfate-polyacrylamide gel followed by transferred to a nitrocellulose membrane (Life Technologies, Carlsbad, CA, USA). The nitrocellulose membrane was incubated with polyclonal rabbit anti-human RASAL2 $(1: 3,000)$ and alkaline phosphatase-conjugated goat anti-rabbit antibody, respectively. After incubation the nitrocellulose membrane was evaluated by ECL.

Immunofluorescent cell staining assay. MDA-MB-231 cells were seeded at $4 \times 10^{5}$ cells/well in 6 -well culture plates. 

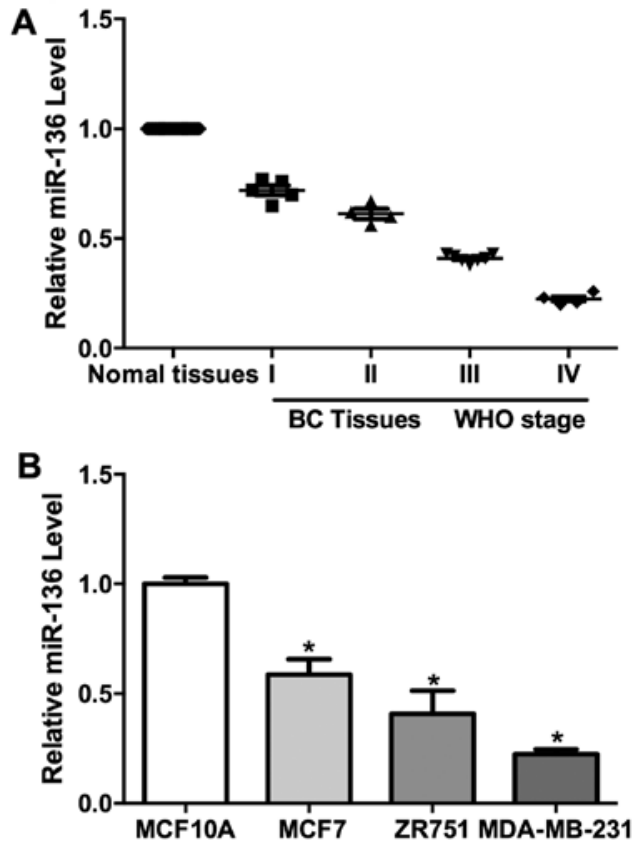

Figure 1. Downregulation of miR-136 in cancer cells and TNBC. (A) miR136 was downregulated in TNBC samples and negatively correlated with pathologic grades (B) miR-136 was downregulated in MCF7, ZR751 and MDA-MB-231 cells compared with MCF10A. Experiments were repeated as least three times independently. Values represent the mean $\pm \mathrm{SD}$. ${ }^{*} \mathrm{P}<0.05$ was calculated using the Student's t-test.

Twenty-four hours later, the attached cells were transfected with $30 \mu \mathrm{M}$ microRNA mimics and allowed to grow further for $72 \mathrm{~h}$. Then the post-treatment cells were trypsinized and re-seeded at a density of $1.5 \times 10^{5}$ cells/well on 8 -mm coverslips in 12-well plates. After additional $48 \mathrm{~h}$, coverslips with cells were fixed in methanol, and probed with primary E-cadherin (BD Biosciences; cat. BD 610182), or vimentin (Santa Cruz Biotechnology; cat. SC-6260) antibodies in 1:100 to 1:1,000, dilution then subsequently with florescent labeled secondary antibodies. Cell nuclei were stained with DAPI. After cell staining the coverslips were mounted with FluorSave reagent (Calbiochem, Darmstadt, Germany). Cells were imaged using Zeiss Meta upright microscope under 63X oil objective.

Statistical analysis. Student's t-test was performed to analyze the significance of differences between the sample means obtained from three independent experiments. One-way ANOVA was used for multiple group comparisons. Differences were considered statistically significant at $\mathrm{P}<0.05$.

\section{Results}

miR-136 expression is downregulated in TNBC and negatively associated with the WHO grades. qPCR analysis revealed that miR-136 transcripts were reduced in tumor tissues compared to normal tissues (Fig. 1A). Further analysis showed that the miR-136 expression were negatively correlated with the WHO grades (Fig. 1A). We also determined miR-136 levels in the immortalized mammary epithelial cells (MCF10A) and three breast cancer cell lines (MCF7, ZR751 and MDA-MB-231). The level of miR-136 is much lower in the TNBC cell lines (MDA-MB-231,ZR751 and MCF7) than control cells (Fig. 1B).
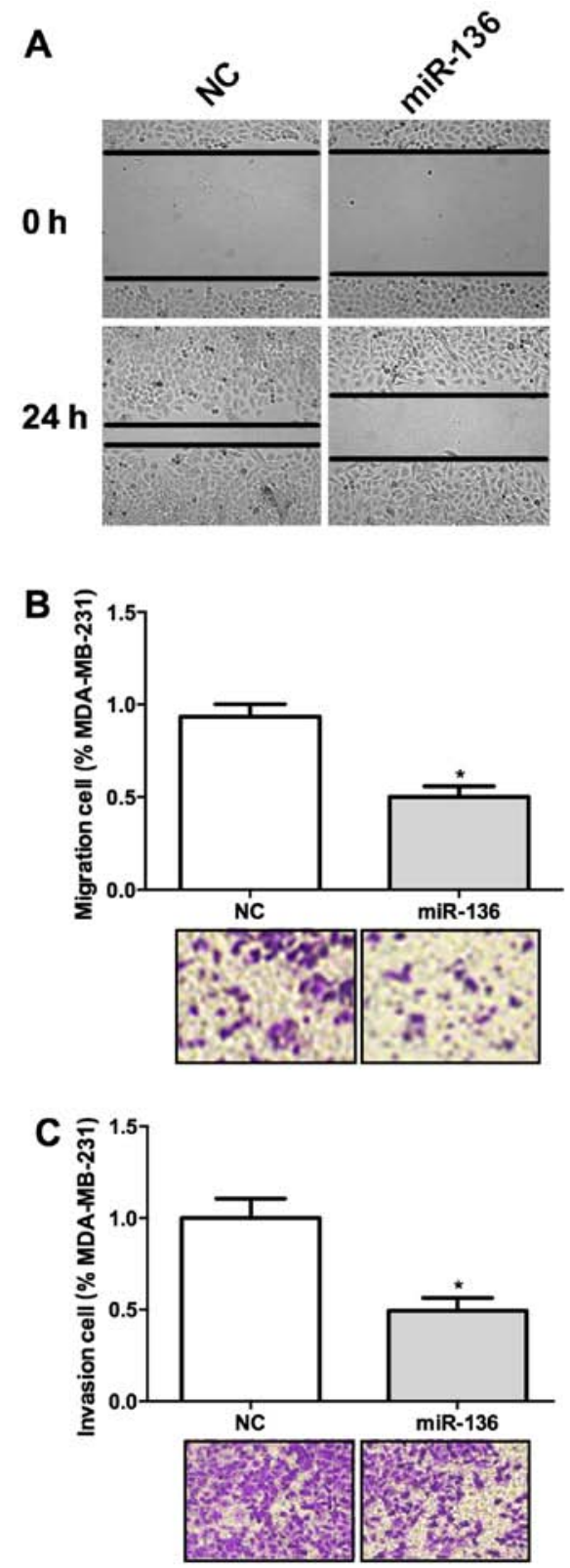

Figure 2. miR-136 represses cell migration and invasion in TNBC cells (A) Overexpression of miR-136 significantly hampered the migration of these cells. (B) miR-136 overexpression decreased the migration ability of MDA-MB-231 cells. (C) MDA-MB-231 cells transfected with miR-136 showed a decreased ability of invasion. Experiments were repeated as least three times independently. Values represent the mean $\pm \mathrm{SD}$. ${ }^{*} \mathrm{P}<0.05$ was calculated using the Student's t-test.

However, the miR-136 expression is negatively correlated with the invasive ability of these breast cancer cell lines (Fig. 1B). These results indicated a role for miR-136 in the development and/or metastasis of TNBC.

miR-136 suppresses cell migration and invasion. We have shown that miR-136 expression was negatively correlated with WHO grade in TNBC, we wonder whether it was involved in the invasion of this cancer. Wound healing assay result suggested that overexpression of miR-136 significantly hampered the migration of these cells (Fig. 2A). Transwell assay also revealed that miR-136 overexpression decreased the migration ability of MDA-MB-231 cells (Fig. 2B). We used 
A

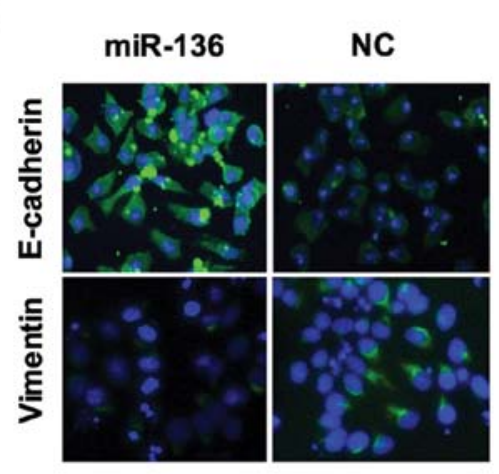

C

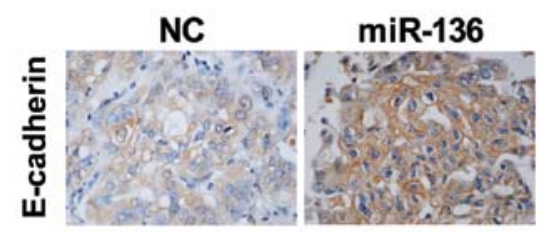

B

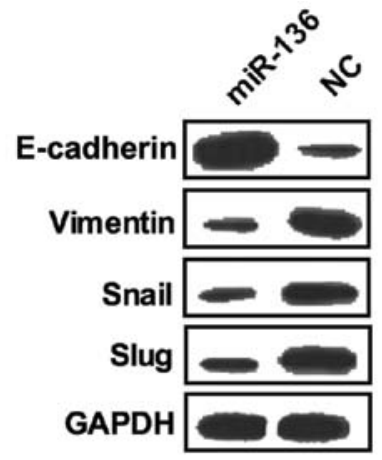

D

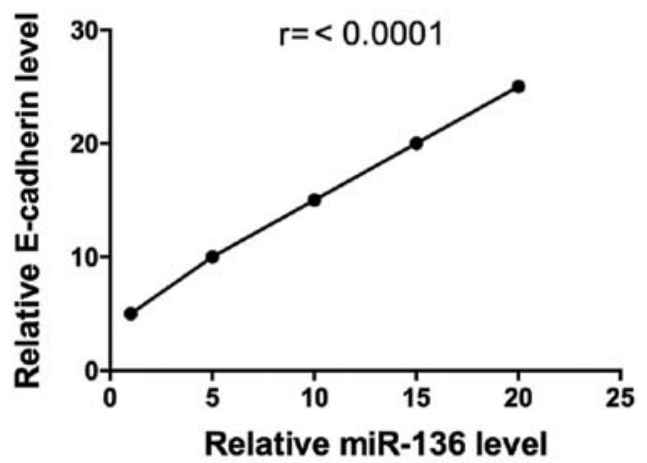

Figure 3. miR-136 functions as a suppressor of EMT of TNBC cells. The representative images of confocal immunofluorescence staining of E-cadherin and vimentin (A), western blot analysis of E-cadherin, vimentin, Smail and SLAG (B). (C) E-cadherin expression were significant higher in miR-136 group than the control. (D) The level of E-cadherin expression were correlated with the injection dose of miR-136 mimics. Experiments were repeated as least three times independently. Values represent the mean $\pm \mathrm{SD}$. The differences were assessed by one way ANOVA.

\section{A}

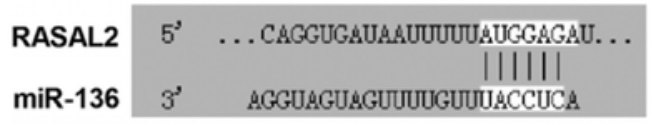

C
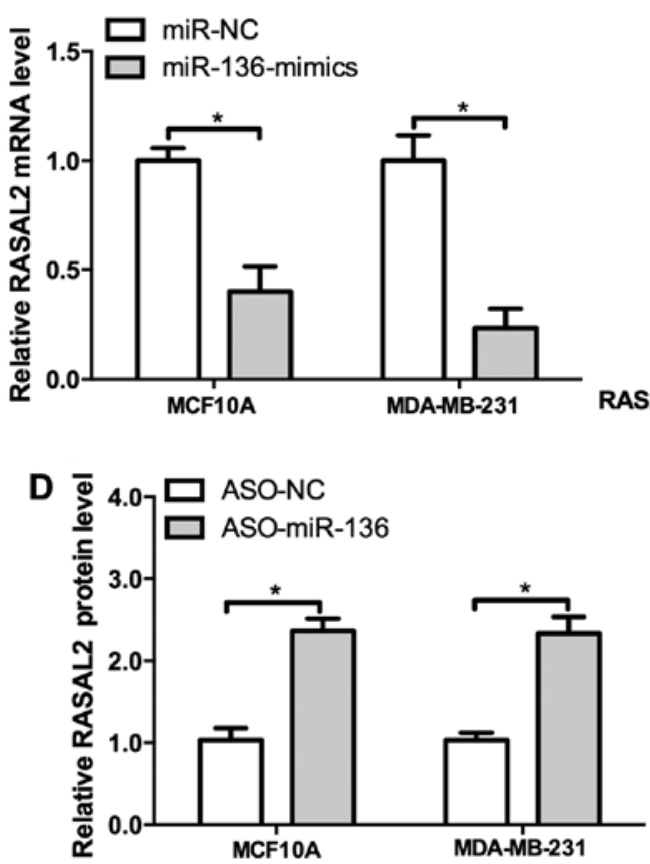

B

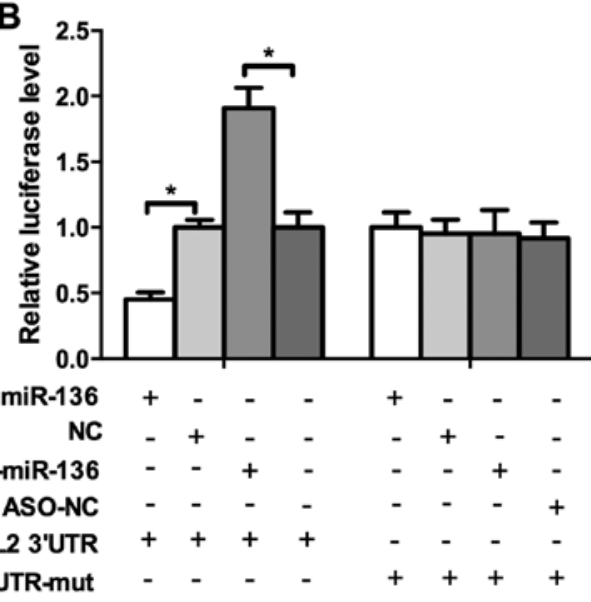

E

RASAL2

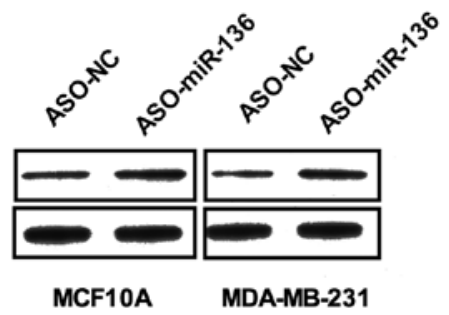

Figure 4. RASAL2 is a target of miR-136. (A) Sequence alignment of miR-136 and the 3'UTR of RASAL2 mRNA. (B) miR-136 overexpression repressed, whereas the ASO elevated the luciferase activities. (C) miR-136 mimics significantly decreased the mRNA level of RASAL2 and conversely, the ASO increased it (D). (E) Western blot analysis of RASAL2. Experiments were repeated as least three times independently. Values represent the mean \pm SD. ${ }^{*} \mathrm{P}<0.05$ was calculated using the Student's t-test. 

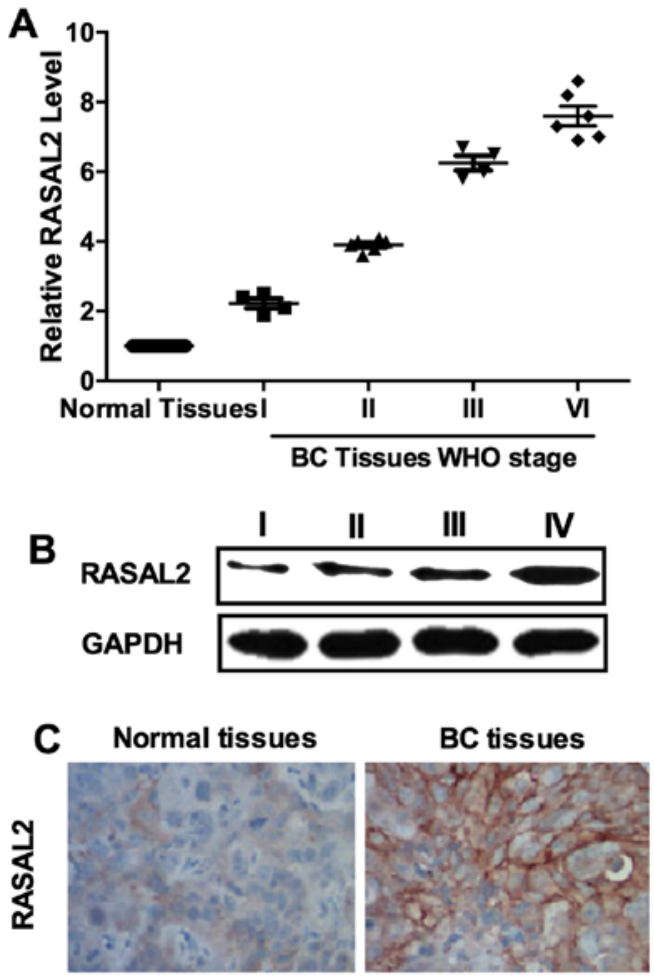

Figure 5. RASAL2 was upregulated in TNBC. (A) RASAL2 expression was upregulated in TNBC samples compared to normal adjacent tissues. (B) Western blot analysis of RASAL2 protein levels in TNBC samples with different pathological grades. (C) Representative images of IHC analysis of RASAL2 expression in TNBC tissues and normal adjacent tissues. Experiments were repeated as least three times independently. Values represent the mean $\pm \mathrm{SD}$.

a Matrigel coated Transwell assay system in vivo to further test the function of miR-136 in cancer invasion. MDA-MB231 cells transfected with miR-136 showed a decreased ability of invasion through the Matrigel (Fig. 2C). These results suggested that miR-136 may act as a suppressor of tumor invasion.

miR-136 suppresses EMT in breast cancer. Epithelial-tomesenchymal transition (EMT) is a critical step for metastatic dissemination (26) thus, we hypothesized that miR-136 may affect EMT. As shown, the epithelial marker E-cadherin fluorescence was visibly enhanced in cells transfected with miR-136 compared to those transfected with scramble miRNA (Fig. 3A). Consistently, the result of confocal imaging analysis indicated that mesenchymal marker vimentin expression was weakened by overexpression of miR-136 (Fig. 3A). Further assessment of a panel of EMT-related genes by western blot analysis showed that, miR-136 treatment induced the expression of E-cadherin and decreased the expression of vimentin, Snail and SLUG, confirmed the results of immunofluorescence assay (Fig. 3B). To further confirmed these results, we established a mouse xenograft model by injecting MDA-MB-231 cells into the mammary gland fat pads. miR-136 mimics and its negative control miRNA were injected into the tumor every 2 days after day 7 of the graft. In miR-136 overexpressed group, E-cadherin expression were significant higher than the control group (Fig. 3C), and the levels of E-cadherin were correlated with the injection dose of miR-136 mimics (Fig. 3D). These results elucidated that miR-136 is a suppressor of EMT in the TNBC cell line.

miR-136 directly regulates RASAL2 expression in breast cancer. Previously we showed that miR-136 may act as tumor suppressor of TNBC metastasis, then we attempted to uncover the underlying mechanisms. RASAL2 is a newly identified cancer-promoting gene in TNBC and it drives mesenchymal invasion and metastasis (14). We investigated the regulatory sequence of miR-136 in the 3'UTR of RASAL2 mRNA. Indeed, RASAL2 harbors a binding site of miR-136 (Fig. 4A). The result of luciferase activity detection revealed miR-136 overexpression repressed, whereas the ASO elevated the luciferase activities (Fig 4B, left panel). Then we mutated the binding site of miR-136 in the 3'UTR of RASAL2 mRNA and the regulatory effect of the mimics or ASO could not be observed (Fig. 4B, right panel). To further confirm these results, the miR-136 mimics, ASO and their negative control were co-transfected into MCF10A and MDA-MB-231 cells. miR-136 mimics significantly decreased the mRNA level of RASAL2 and conversely, the ASO increased it (Fig. 4C and D). Western blot analysis confirmed these results (Fig. 4E). Conclusively, those results demonstrated that RASAL2 is a direct target of miR-136.

RASAL2 is upregulated in TNBC. Our results showed that RASAL2 was negatively regulated by miR-136 which functioned as a tumor suppressor in TNBC. Thus, we inferred that RASAL2 may be a cancer-promoting gene. We determined the expression of RASAL2 in 20 samples of TNBC tissues and their normal adjacent tissues (NAT) by qPCR. The results showed that RASAL2 was unregulated in TNBC tissues and its levels were correlated with WHO grade 90 (Fig. 5A). Western blot analysis and IHC results were used as further confirmation (Fig. 5B and C). These results supported that RASAL2 may function as an oncogene in TNBC.

miR-136 suppresses breast cell migration and invasion through RASAL2. We identified miR-136 as a tumor suppressor of TNBC, and RASAL2 may served as its target. Whether miR-136 regulate cell invasion through RASAL2 remains unclear. To validate this hypothesis, we co-transfected miR-136 mimics and RASAL2 into MCF10A and MDA-MB-231 cells. Compared with control, miR-136 suppressed RASAL2 expression both at mRNA and protein levels (Fig. 6A and B). Moreover, RASAL2 restoration significantly elevated the migration and invasion of MDA-MB-231 cells which were inhibited by miR-136 (Fig. 6C and D), indicating that RASAL2 could rescue miR-136 mediated suppression of migration and invasion of TNBC cells. These results suggested that RASAL2 may be a functional target of miR-136 in TNBC cell invasion.

\section{Discussion}

Breast cancer is an extremely heterogeneous disease, comprising a number of different subtypes. TNBC, representing only $10-24 \%$ of all breast cancers diagnosed, have been recently intensely investigated because of their aggressive clinical behavior. Patients with TNBC are often of 

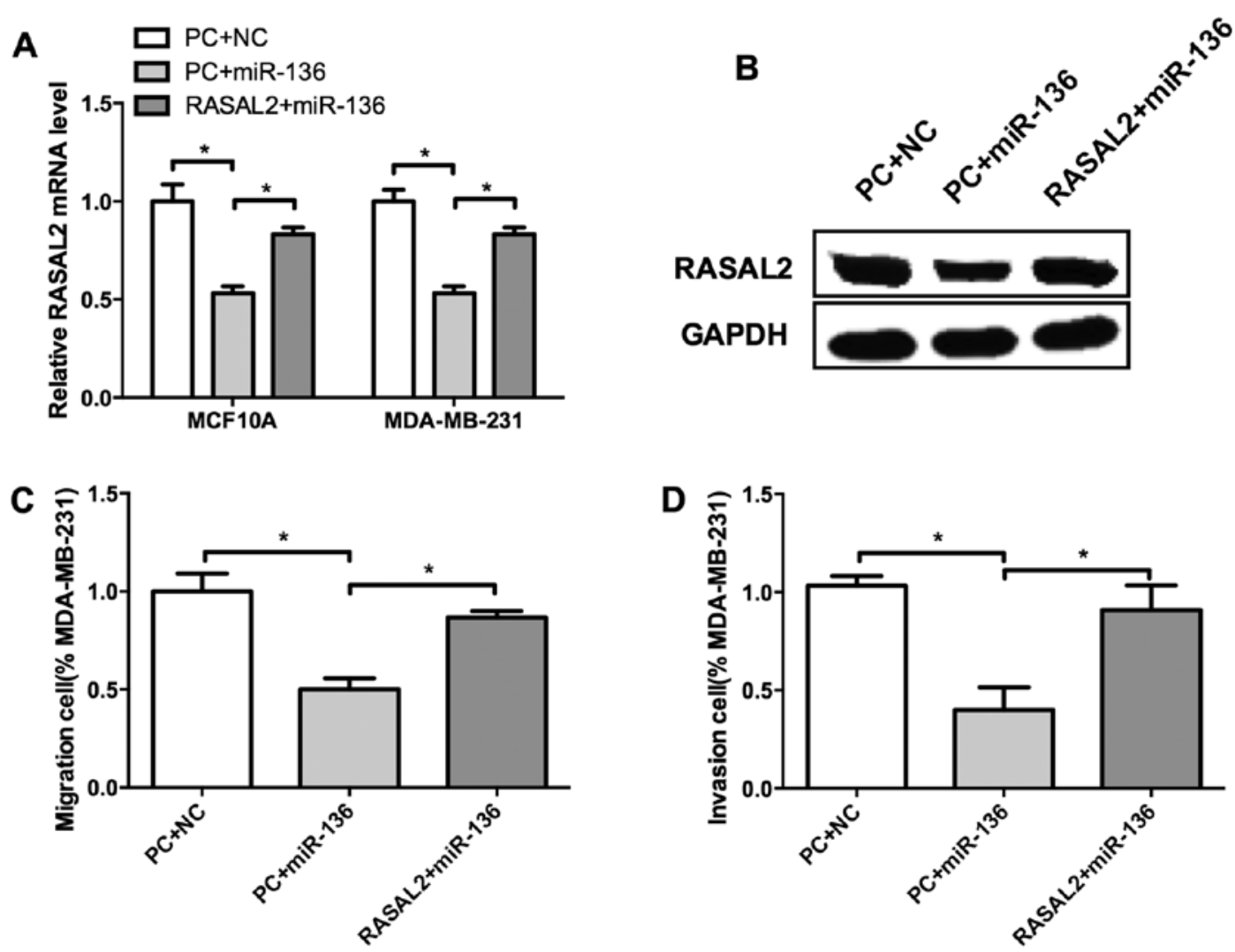

Figure 6. miR-136 suppresses breast cell migration and invasion through RASAL2. Compared with control, miR-136 suppressed RASAL2 expression levels of both mRNA (A) and protein (B). RASAL2 restoration significantly elevated the migration (C) and invasion (D) of MDA-MB-231 cells which were inhibited by miR-136. Experiments were repeated as least three times independently. Values represent the mean \pm SD. "P<0.05 was calculated using the Student's t-test.

younger age, and tend to develop tumors of larger size, and have an increased risk of distant metastasis and death within 5 years $(27,28)$. However, the lack of understanding of the underlying mechanisms of distant metastasis and early relapse impedes new treatment modality development for TNBC. In the present study, we identified the microRNA, miR-136, as an tumor suppressor of TNBC and demonstrated that it functioned through downregulating RASAL2, a newly identified cancer-promoting gene in TNBC. This study adds to our understanding of the underlying mechanism of TNBC invasion and metastasis.

RASAL2 was first identified as an tumor and metastasis suppressor in breast cancer (10). However, a more precise analysis taking the gene subtype into consideration showed that RASAL2 played a pro-oncogenic role in TNBC, rather as a tumor suppressive RAS-GAP protein in luminal tumors (the most commonly diagnosed breast cancer subtype) $(14,29)$. In a Kaplan-Meier meta-analyses consisting of 1,789 patients, RASAL2 expression level was shown as not prognostic in unselected patients, but an expression level in the top 30\% was significantly correlated with the poor prognosis in patients with basal tumors, which overlap largely with TNBC tumors (14). Our results showed that RASAL2 was markedly

upregulated in high-grade TNBC, consistent with other largescale analyses (12-14). This result may add new evidence to support RASAL2 as a novel prognostic biomarker of patients with TNBC.
miR-136 was previously shown to be tightly associated with tumorigenesis and metastasis. It was reported to act as a cancer-promoting gene in human NSCLC (24), and may also act as a tumor suppressor in human glioma (25). Our results showed that it was downregulated in TNBC and negative correlated with the WHO grades. It repressed cell migration and invasion in vitro and restrained the EMT process in a xenograft mouse model. These results provide new evidence for miR-136 to be a tumor suppressor specially in the process of invasion and metastasis of TNBC. miR-136 was recently reported to be involved in the drug resistance of human epithelial ovarian cancer and glioma $(30,31)$. Future studies are needed to verify whether miR-136 regulates sensitivity of chemotherapeutics for patients diagnosed with TNBC.

In conclusion, our findings demonstrated that miR-136 was marked downregulated while RASAL2 was upregulated in TNBC and both were significantly correlated with clinical stage. Furthermore, our results suggested miR-136 suppressed cell migration and invasion as well as the EMT process in breast cancer. Moreover, RASAL2 harbors a binding site of miR-136 and overexpression of miR-136 significantly decreased the mRNA level of RASAL2, which indicated miR-136 may directly regulate RASAL2 expression in the development of breast cancer. These results may validate a pathogenetic role of miR-136 in TNBC and establish a potential regulatory mechanism involving miR-136/RASAL2/ EMT in TNBC. 


\section{Acknowledgements}

The present study was supported by the Department of Pathology, Harbin Medical University.

\section{References}

1. Torre LA, Bray F, Siegel RL, Ferlay J, Lortet-Tieulent J and Jemal A: Global cancer statistics, 2012. CA Cancer J Clin 65: 87-108, 2015.

2. Autier P, Boniol M, La Vecchia C, Vatten L, Gavin A, Héry C and Heanue M: Disparities in breast cancer mortality trends between 30 European countries: Retrospective trend analysis of WHO mortality database. BMJ 341: c3620, 2010.

3. Foulkes WD, Smith IE and Reis-Filho JS: Triple-negative breast cancer. N Engl J Med 363: 1938-1948, 2010.

4. Kumar P and Aggarwal R: An overview of triple-negative breast cancer. Arch Gynecol Obstet 293: 247-269, 2015

5. Lee J and Gollahon L: Nek2-targeted ASO or siRNA pretreatment enhances anticancer drug sensitivity in triple-negative breast cancer cells. Int J Oncol 42: 839-847, 2013.

6. Berrada N, Delaloge S and André F: Treatment of triple-negative metastatic breast cancer: Toward individualized targeted treatments or chemosensitization? Ann Oncol 21 (Suppl 7): vii30-vii35, 2010.

7. Downward J: Targeting RAS signalling pathways in cancer therapy. Nat Rev Cancer 3: 11-22, 2003.

8. Pylayeva-Gupta Y, Grabocka E and Bar-Sagi D: RAS oncogenes: Weaving a tumorigenic web. Nat Rev Cancer 11: 761-774, 2011.

9. Bos JL, Rehmann H and Wittinghofer A: GEFs and GAPs: Critical elements in the control of small G proteins. Cell 129: 865-877, 2007

10. McLaughlin SK, Olsen SN, Dake B, De Raedt T, Lim E, Bronson RT, Beroukhim R, Polyak K, Brown M, Kuperwasser C, et al: The RasGAP gene, RASAL2, is a tumor and metastasis suppressor. Cancer Cell 24: 365-378, 2013.

11. Xu Y, Deng Y, Ji Z, Liu H, Liu Y, Peng H, Wu J and Fan J: Identification of thyroid carcinoma related genes with mRMR and shortest path approaches. PLoS One 9: e94022, 2014.

12. Huang $\mathrm{Y}$, Zhao M, Xu H, Wang $\mathrm{K}, \mathrm{Fu} \mathrm{Z}$, Jiang $\mathrm{Y}$ and $\mathrm{Yao} \mathrm{Z}$ : RASAL2 down-regulation in ovarian cancer promotes epithelialmesenchymal transition and metastasis. Oncotarget 5: 6734-6745, 2014.

13. Li N and Li S: RASAL2 promotes lung cancer metastasis through epithelial-mesenchymal transition. Biochem Biophys Res Commun 455: 358-362, 2014.

14. Feng M, Bao Y, Li Z, Li J, Gong M, Lam S, Wang J, Marzese DM, Donovan N, Tan EY, et al: RASAL2 activates RAC1 to promote triple-negative breast cancer progression. J Clin Invest 124: 5291-5304, 2014.

15. Zhang Y, Yang P and Wang XF: Microenvironmental regulation of cancer metastasis by miRNAs. Trends Cell Biol 24: 153-160, 2014.
16. Pencheva $\mathrm{N}$ and Tavazoie SF: Control of metastatic progression by microRNA regulatory networks. Nat Cell Biol 15: 546-554, 2013.

17. Calin GA and Croce CM: MicroRNA signatures in human cancers. Nat Rev Cancer 6: 857-866, 2006.

18. Nicoloso MS, Spizzo R, Shimizu M, Rossi S and Calin GA: MicroRNAs - the micro steering wheel of tumour metastases. Nat Rev Cancer 9: 293-302, 2009.

19. Valastyan S, Reinhardt F, Benaich N, Calogrias D, Szász AM, Wang ZC, Brock JE, Richardson AL and Weinberg RA: A pleiotropically acting microRNA, miR-31, inhibits breast cancer metastasis. Cell 137: 1032-1046, 2009.

20. Yu F, Yao H, Zhu P, Zhang X, Pan Q, Gong C, Huang Y, Hu X, $\mathrm{Su}$ F, Lieberman J, et al: let-7 regulates self renewal and tumorigenicity of breast cancer cells. Cell 131: 1109-1123, 2007.

21. Liu X, Sempere LF, Ouyang H, Memoli VA, Andrew AS, Luo Y, Demidenko E, Korc M, Shi W, Preis M, et al: MicroRNA-31 functions as an oncogenic microRNA in mouse and human lung cancer cells by repressing specific tumor suppressors. J Clin Invest 120: 1298-1309, 2010.

22. Yu J, Wang F, Yang GH, Wang FL, Ma YN, Du ZW and Zhang JW: Human microRNA clusters: Genomic organization and expression profile in leukemia cell lines. Biochem Biophys Res Commun 349: 59-68, 2006.

23. Lee DY, Jeyapalan Z, Fang L, Yang J, Zhang Y, Yee AY, Li M, Du WW, Shatseva T and Yang BB: Expression of versican 3'-untranslated region modulates endogenous microRNA functions. PLoS One 5: e13599, 2010.

24. Shen S, Yue H, Li Y, Qin J, Li K, Liu Y and Wang J: Upregulation of miR-136 in human non-small cell lung cancer cells promotes Erk1/2 activation by targeting PPP2R2A. Tumour Biol 35: 631-640, 2014.

25. Yang Y, Wu J, Guan H, Cai J, Fang L, Li J and Li M: MiR-136 promotes apoptosis of glioma cells by targeting AEG-1 and Bcl-2. FEBS Lett 586: 3608-3612, 2012.

26. Chaffer CL and Weinberg RA: A perspective on cancer cell metastasis. Science 331: 1559-1564, 2011.

27. Podo F, Buydens LM, Degani H, Hilhorst R, Klipp E, Gribbestad IS, Van Huffel S, van Laarhoven HW, Luts J, Monleon D, et al; FEMME Consortium: Triple-negative breast cancer: Present challenges and new perspectives. Mol Oncol 4: 209-229, 2010

28. Carey L, Winer E, Viale G, Cameron D and Gianni L: Triplenegative breast cancer: Disease entity or title of convenience? Nat Rev Clin Oncol 7: 683-692, 2010.

29. Aysola K, Desai A, Welch C, Xu J, Qin Y, Reddy V, Matthews R, Owens C, Okoli J, Beech DJ, et al: Triple negative breast cancer: An overview. Hereditary Genet 2013 (Suppl 2): 2013, 2013.

30. Zhao H, Liu S, Wang G, Wu X, Ding Y, Guo G, Jiang J and Cui S: Expression of miR-136 is associated with the primary cisplatin resistance of human epithelial ovarian cancer. Oncol Rep 33: 591-598, 2015.

31. Wu H, Liu Q, Cai T, Chen YD, Liao F and Wang ZF: MiR-136 modulates glioma cell sensitivity to temozolomide by targeting astrocyte elevated gene-1. Diagn Pathol 9: 173, 2014 